\title{
The Role of Platelets in the
} Stimulation of Neuronal Synaptic Plasticity, Electric Activity, and Oxidative Phosphorylation: Possibilities for New Therapy of Neurodegenerative Diseases

\author{
Ekaterina Kopeikina and Eugene D. Ponomarev* \\ School of Biomedical Sciences, Faculty of Medicine, The Chinese University of Hong Kong, Shatin, Hong Kong
}

OPEN ACCESS

Edited by:

Eleonora Vannini,

Institute of Neuroscience, National

Research Council (CNR), Italy

Reviewed by:

Federico Carlini,

Università di Genova, Italy

llias Kazanis,

University of Cambridge,

United Kingdom

Zeki llkan,

University of Oxford, United Kingdom

*Correspondence:

Eugene D. Ponomarev

eponomarev@cuhk.edu.hk

Specialty section:

This article was submitted to

Cellular Neuropathology,

a section of the journal

Frontiers in Cellular Neuroscience

Received: 13 March 2021 Accepted: 23 June 2021

Published: 14 July 2021

Citation:

Kopeikina E and Ponomarev ED (2021) The Role of Platelets in the Stimulation of Neuronal Synaptic

Plasticity, Electric Activity, and

Oxidative Phosphorylation:

Possibilities for New Therapy of

Neurodegenerative Diseases.

Front. Cell. Neurosci. 15:680126. doi: 10.3389/fncel.2021.680126
The central nervous system (CNS) is highly vascularized where neuronal cells are located in proximity to endothelial cells, astroglial limitans, and neuronal processes constituting integrated neurovascular units. In contrast to many other organs, the CNS has a blood-brain barrier (BBB), which becomes compromised due to infection, neuroinflammation, neurodegeneration, traumatic brain injury, and other reasons. BBB disruption is presumably involved in neuronal injury during epilepsy and psychiatric disorders. Therefore, many types of neuropsychological disorders are accompanied by an increase in BBB permeability leading to direct contact of circulating blood cells in the capillaries with neuronal cells in the CNS. The second most abundant type of blood cells are platelets, which come after erythrocytes and outnumber $\sim 100$-fold circulating leukocytes. When BBB becomes compromised, platelets swiftly respond to the vascular injury and become engaged in thrombosis and hemostasis. However, more recent studies demonstrated that platelets could also enter CNS parenchyma and directly interact with neuronal cells. Within CNS, platelets become activated by recognizing major brain gangliosides on the surface of astrocytes and neurons and releasing a milieu of pro-inflammatory mediators, neurotrophic factors, and neurotransmitters. Plateletderived factors directly stimulate neuronal electric and synaptic activity and promote the formation of new synapses and axonal regrowth near the site of damage. Despite such active involvement in response to CNS damage, the role of platelets in neurological disorders was not extensively studied, which will be the focus of this review.

Keywords: platelets, neurons, neurodegeneration, major brain gangliosides, serotonin, CNS repair

\section{INTRODUCTION}

Platelet biology recently gains a particular interest in neuroscience. Quite intriguing that platelet granules, which are organized like vesicles of presynaptic neurons, store key neurotransmitters: dopamine, glutamate, histamine, serotonin, ATP along with proper receptors and transporters for these molecules (Rainesalo et al., 2005). Moreover, platelet granules contain a 
significant set of neurotrophic factors such as EGF, NGF and, BDNF (Yamamoto and Gurney, 1990; Au et al., 2014; Kniewallner et al., 2014). Finally, platelets and the CNS might be linked via specific structural similarity of platelets with neurons. Both cell types have complex granule organization with diverse content and regulated secretion, as well as the presence of mitochondria and extensive oxidative phosphorylation. Platelet granule secretion and neuronal synaptic release work virtually identically engaging the same triggers and downstream signaling cascades (Ponomarev, 2018). Platelets also contain a variety of pro-inflammatory factors that could strongly influence the pathology of neurodegenerative diseases (Reed et al., 2000). Nevertheless, the platelet role in neuroscience remains neglected. This review aims to elucidate an important role of platelets in most common neurologic disorders: epilepsy, traumatic brain injury, Alzheimer's disease, multiple sclerosis, and Parkinson's disease. Future perspectives to use platelets and antiplatelet drugs for diagnostics and treatment of neurologic disorders will be also discussed.

\section{EPILEPSY AND TRAUMATIC BRAIN INJURY}

Platelets were found in our very recent study (Kopeikina et al., 2020) to actively participate in the development of several epilepsy-associated pathological events in the CNS such as the swift release of serotonin, direct stimulation of neuronal electric activity, blood-brain barrier (BBB) permeabilization, release, and induction of expression of pro-inflammatory mediators, and induction of neuronal oxidative stress (Kopeikina et al., 2020). Previously we found that platelets were also actively involved in TBI pathology (Dukhinova et al., 2018). These findings support the hypothesis that platelets play a significant role in epilepsy development, especially in epilepsy that is associated with TBI.

Epilepsy is a neurological disorder that affects more than 70 million globally and is manifested by the occurrence of seizures due to global or focal abnormally high brain electric activity (Thijs et al., 2019). This disorder is also associated with motor, cognitive, and psychological abnormalities (Devinsky et al., 2018). Although the real cause of epilepsy is unknown, some factors create a predisposition to this disease. Previous studies on epilepsy have mostly focused on genetic factors and pathological events related to neuronal functions in the central nervous system (CNS). Such studies reported that the imbalance between activating and inhibitory circuits in the area of abnormal neuronal electric activity plays a major role in seizure development (Staley, 2015). Blocking inhibitory $\mathrm{GABA}_{\mathrm{A}}$ receptors with pharmacological antagonists, such as pentylenetetrazole (PTZ), results in seizure development in several species, including humans and mice. The acute PTZ-induced seizure model is widely used to study the effects of new antiepileptic drugs (Löscher, 2011). Stimulation of activating glutamate receptors with specific agonists also results in epileptic seizures (McKhann et al., 2003). Available antiepileptic drugs mostly change the balance toward inhibitory pathways. Yet more than $30 \%$ of epilepsy patients do not respond to these drugs (Thijs et al., 2019). Thus, it is critical to find new drugs to treat epilepsy based on other mechanisms and possibly targeting other cell types besides neurons.

Although the main cause for epilepsy is known, a predisposition to this disorder comes from both genetic and environmental factors such as abnormal CNS development and CNS injuries such as stroke and traumatic brain injury (TBI; Devinsky et al., 2018; Thijs et al., 2019). As many as $~ 50 \%$ of all epilepsy cases are triggered by initial neuronal injury and are classified as acquired epilepsy (AE). The three stages of AE include: (1) initial neuronal injury, (2) epileptogenesis, and (3) chronic epilepsy (period of spontaneous recurrent seizures). TBI and stroke are the most frequent brain injuries that often result in the development of $\mathrm{AE}$. AE seizures are classified as acute (hours or days post-TBI) and chronic (from weeks to months). The occurrence of seizures following TBI or other insult is classified as immediate (less than $24 \mathrm{~h}$ ), early (1-7 days), or late (more than 1 week; Tomkins et al., 2008; Lucke-Wold et al., 2015; Glushakov et al., 2016) AE.

Neuronal functions are substantially influenced by a milieu of platelet-derived factors. One of our key results of whole-genome transcriptome profiling followed by real-time RT PCR validation is that platelets induced expression of many genes related to neuronal electric/synaptic activity, neuroinflammation, and oxidative phosphorylation in TBI and epilepsy models (Dukhinova et al., 2018; Kopeikina et al., 2020). Platelets induced the expression of mRNA for pro-inflammatory cytokines $I L-1 B, I L-6$, and TNF (Kopeikina et al., 2020). The oxidative phosphorylation pathway (e.g., expression of mRNA for mitochondrial genes MT-CO1, MT-ATP6, and $M T$-ND6) was also significantly upregulated, while the glycolysis pathway is downregulated (Kopeikina et al., 2020). Platelets also upregulated the expression of mRNA for several early response genes responsible for neuronal synaptic plasticity, such as PSD95, TrkB, Syn1, FOSB, EGR1, ARC. We also found that platelets stimulated the formation of dendritic spines and new synapses both in vitro and in vivo (Dukhinova et al., 2018; Kopeikina et al., 2020).

Serotonin $(5 \mathrm{HT})$ is known to contribute to thrombosis, but at the same time, this neurotransmitter is involved in the regulation of innate and adaptive immune responses, neuroinflammation, anaphylaxis, and CNS tissue repair (Sotnikov et al., 2013; Starossom et al., 2015; Dukhinova et al., 2018; Kopeikina et al., 2020). During TBI, we showed that platelet-derived 5HT enhances neuronal axonal growth and the formation of new synapses (Dukhinova et al., 2018). We also demonstrated the significant role of platelets and platelet-derived 5HT in the development of PTZ-induced seizures in mice (Gharedaghi et al., 2014; Carhart-Harris and Nutt, 2017). Our in vitro and in vivo experiments where we did the co-incubation of platelets with brain slices, or with cultured neurons, or adoptive transfer of serotonin-depleted platelets strongly proved that plateletderived 5HT was required to increase neuronal electric activity (Dukhinova et al., 2018; Kopeikina et al., 2020).

Quite interesting that in both PTZ-induced epilepsy and TBI models, we observed increased neuronal electric activity, 
and expression of pro-inflammatory and synaptic plasticity genes (Dukhinova et al., 2018; Kopeikina et al., 2020). Remarkable, intracranial injection of a small volume of platelets, but not saline or platelet-poor plasma, induced severe seizures and epilepsy-like elevated neuronal electrical activity (Kopeikina et al., 2020), which bridge together TBI and epilepsy and may imply the mechanisms of development of acute AE such as post-traumatic or post-stroke epilepsy. An elevated level of oxidative phosphorylation often results in ROS formation leading to oxidative stress in the CNS (Pearson-Smith and Patel, 2017), which was confirmed in our studies where platelets upregulated a large number of mitochondria oxidative phosphorylation genes leading to reactive oxygen species (ROS) formation in neurons leading to neuronal oxidative stress in vitro and in vivo (Kopeikina et al., 2020).

Thus, we hypothesize that platelets could promote the development of immediate (less than $24 \mathrm{~h}$ ) AE by directly stimulating neuronal electric activity by robust $5 \mathrm{HT}$ release (Kopeikina et al., 2020). Platelets could also stimulate the development of early AE (1-7 days) possibly by upregulation of the number of genes related to neuronal activity and synaptic plasticity, neuroinflammation, and oxidative phosphorylation (Dukhinova et al., 2018; Kopeikina et al., 2020). Finally, platelets could contribute to late $\mathrm{AE}$ (more than 1 week) by stimulating axonal regrowth and formation of new synapses in the area around brain injury (Dukhinova et al., 2018), which might lead to the imbalance of excitatory vs. inhibitory circuits (Musto et al., 2016; Pfisterer et al., 2020).

Our studies indicated a new concept that during epilepsy and TBI platelets could enter CNS due to increased BBB permeability and could interact with neuronal cells via cell-cell contacts and/or via secretion of soluble factors. In Table 1, we summarized the role of platelets in epilepsy and TBI. During initial CNS insult such as epileptic seizures or TBI, BBB permeability increases and platelets enter CNS perivascular space where they interact with astroglial and neuronal lipid rafts (Sotnikov et al., 2013) and secrete neurotransmitters (serotonin; Kopeikina et al., 2020), cytokines (IL-1 $\alpha$; Sotnikov et al., 2013; Starossom et al., 2015), chemokines (platelet factor 4, PF4; Starossom et al., 2015) and lipid mediators (platelet-activating factor, PAF; thromboxane, etc.; Dukhinova et al., 2018). At this stage, platelets contribute to the development of neuroinflammation leading to further increase in BBB permeability (Kopeikina et al., 2020) and stimulating macroglia activation and leukocyte infiltration from the periphery (Sotnikov et al., 2013; Starossom et al., 2015; Dukhinova et al., 2018; Ponomarev, 2018). Increased levels of neuroinflammation result in a further increase in $\mathrm{BBB}$ permeability. At this stage platelets enter CNS parenchyma

TABLE 1 | Platelet-neural crosstalk and possible mechanisms involved in the development of neurodegenerative diseases and central nervous system (CNS) repair ${ }^{1}$.

\begin{tabular}{llll}
\hline $\begin{array}{l}\text { Stage of } \\
\text { neuro-pathology }\end{array}$ & $\begin{array}{l}\text { Blood-brain barrier } \\
\text { (BBB) permeability }\end{array}$ & $\begin{array}{l}\text { Recognition of major brain gangliosides on } \\
\text { astroglial and neuronal lipid rafts and } \\
\text { secretion of platelet-derived factors }\end{array}$ & $\begin{array}{l}\text { Effect of platelets on CNS function } \\
\text { (Role of platelet-derived factors) }\end{array}$ \\
\hline Normal CNS & Intact & No effect \\
\hline $\begin{array}{l}\text { Primary CNS } \\
\text { damage }\end{array}$ & Mild BBB permeability & $\begin{array}{l}\text { Platelets enter CNS perivascular space, } \\
\text { recognize major brain gangliosides on astroglial }\end{array}$
\end{tabular}
lipid rafts, and secrete serotonin/5-HT, BDNF, PAF, PF4, and IL-12, 3

Platelets initiate neuroinflammation by stimulating perivascular macrophages and microglia (PAF, PF4, IL-1) ${ }^{2-6}$ Platelets promote blood coagulation (5-HT, ATP $)^{4,6}$

Secondary CNS Severe BBB permeability
damage
major brain gangliosides on neuronal lipid rafts, PAF, PF4, and IL-12-6
Platelets enter CNS parenchyma, recognize and continue to secrete serotonin/5-HT, BDNF,

Platelets stimulate neuronal synaptic activity $(5-\mathrm{HT}, \mathrm{BDNF})^{4,6}$

Platelets stimulate neuronal electric activity $(5-H T)^{4,6}$

Platelets induce mitochondrial oxidative phosphorylation and oxidative stress in neurons (unknown platelet-derived factor $)^{6}$

\begin{tabular}{lll}
\hline CNS repair & $\begin{array}{l}\text { Decreased BBB } \\
\text { permeability }\end{array}$ & $\begin{array}{l}\text { Platelet-derived factors result in marked } \\
\text { changes in CNS gene expression profile }\end{array}$
\end{tabular}

Platelets induce expression of neuronal early response genes (5-HT, BDNF) ${ }^{4,6}$

Platelets induce the formation of dendritic spines and new synapses $(5-\mathrm{HT}, \mathrm{BDNF})^{4}$

Platelets induce axonal outgrowth $(5-\mathrm{HT})^{4}$

\footnotetext{
${ }^{1}$ Proposed scheme based on cited below literature data. ${ }^{2}$ Sotnikov et al. (2013). ${ }^{3}$ Starossom et al. (2015). ${ }^{4}$ Dukhinova et al. (2018). ${ }^{5}$ Ponomarev (2018). ${ }^{6}$ Kopeikina et al. (2020).
} 
and directly interact with neurons by stimulating their electric activity via secretion of serotonin (Kopeikina et al., 2020) and stimulating neuronal gene expression related to synaptic plasticity and oxidative phosphorylation (Dukhinova et al., 2018; Kopeikina et al., 2020). Finally increased expression of neuronal synaptic plasticity genes stimulated the formation of new synapses around the area of injury promoting CNS repair and recovery from the disease (Dukhinova et al., 2018; Table 1). We believe that this concept could be extended to other neurodegenerative diseases, as discussed below.

\section{ALZHEIMER'S DISEASE}

It became quite clear after our recent studies that platelets can stimulate neuronal electric and synaptic activity during traumatic brain injury and epilepsy (Dukhinova et al., 2018; Kopeikina et al., 2020). We believe that similar processes of interaction of platelets with neurons could be found in other types of neurological disorders, such as Alzheimer's disease (AD). AD is a neurodegenerative disorder, which is the common cause of dementia in elderly. Neuropathology includes extracellular $\beta$ Amyloid (A $\beta$ ) plaques co-localized with ganglioside containing neuronal lipid rafts, neuronal intracellular neurofibrillary tau protein tangles, and neuroinflammation (Masters et al., 2015; Dukhinova et al., 2019). Recent studies demonstrated regulation of BBB permeability by circadian rhythms and sleep (Cuddapah et al., 2019), indicating possibilities for platelet migration into CNS during even early stages of various pathological conditions such as $\mathrm{AD}$. To support this hypothesis, it was shown that $\mathrm{A} \beta$ undergoes daily oscillation in interstitial fluid in the vicinity of brain blood vessels, suggesting a possible influx of $A \beta$ from the periphery into CNS (Kress et al., 2018). Very recent studies indicate that platelets can contribute to BBB disruption (Kopeikina et al., 2020; Wu et al., 2021) and can transfer $\mathrm{A} \beta$ from blood vessels into CNS (Wu et al., 2021; Table 1). Moreover, it was demonstrated that activated platelets from APP/PS1 transgenic mice invade brain parenchyma and are closely associated with astrocytes (Kniewallner et al., 2020) that are enriched with major brain gangliosides in lipid rafts of astroglial limitations and efficiently activate platelets (Sotnikov et al., 2013). Platelets and their secreted factors could affect many cell types involved in the regulation of $\mathrm{BBB}$ integrity including endothelial cells, astroglia, and pericytes (Fang et al., 2011; Gonzales et al., 2020; Kniewallner et al., 2020).

Indeed, outside of CNS, platelets are known as the main source of A $\beta$ (Veitinger et al., 2014; Inyushin et al., 2020). Thus, the cellular mechanisms of AD can be effectively studied using platelet sample preparations, due to dramatically increased levels of the $\mathrm{A} \beta$ precursor protein (APP) in them in comparison to all peripheral tissues, and all three isoforms of APP $(130,110$, and $106 \mathrm{kDa}$ ) being detectable within platelets. During platelet activation, full-length APP is cleaved by a $\mathrm{Ca}^{2+}$-dependent cysteine protease, while APP processing is altered in $\mathrm{AD}$ patients when compared to healthy individuals. This results in a decreased ratio between the $130 \mathrm{kDa}$ and $106-110 \mathrm{kDa}$ of cleaved APP isoforms, which implies that APP isoform ratios in platelets might act as a biomarker for AD (Tobergte and Curtis, 2013). A recent study demonstrated that $\mathrm{A} \beta$ fragments could enter CNS from blood vessels in the mouse model of AD (Bu et al., 2018). Using the parabiosis model, where there was connected the blood system of control (wild-type, WT) mice and transgenic mice with human APP overexpression in the CNS, it was revealed that in a previously healthy WT mouse the brain exhibited signs of $\mathrm{AD}$ that include $\mathrm{A} \beta$ depositions, neurodegeneration, and neuroinflammation ( $\mathrm{Bu}$ et al., 2018). Thus, platelets can secrete various processed forms of APP and other substances while infiltrating the brain, which results in the growth of $\mathrm{A} \beta$ depositions in the brain and increases the permeability of BBB (Espinosa-Parrilla et al., 2019; Wu et al., 2021). We demonstrated that major brain gangliosides within neuronal lipid rafts in post-synaptic membranes induced platelets' granule release (Sotnikov et al., 2013) suggesting a possible mechanism of $\mathrm{A} \beta$ secretion by platelets in the CNS. At the same time, brainspecific gangliosides serve as an anchor point for binding of $\mathrm{A} \beta$ peptides, which was critical for $\mathrm{AD}$ development in 5XFAD mouse model (Ponomarev, 2018; Dukhinova et al., 2019).

Besides secretion of $A \beta$ peptides, platelets could also stimulate neuronal electric activity vis production of serotonin (Dukhinova et al., 2018; Kopeikina et al., 2020; Table 1), while neurons with a high level of electric activity were found in close vicinity of $\mathrm{A} \beta$ depositions (Busche et al., 2008; Liu et al., 2018). Thus, we could speculate that platelets contribute to elevated neuronal electric activity in $\mathrm{AD}$. The elevated level of electric activity could even result in the development of seizures in some AD patients (Born, 2015; Kitchigina, 2018). Thus, elevated neuronal activity was shown to contribute to $\mathrm{AD}$ pathology via multiple mechanisms (Hefter et al., 2020), while our study indicated the critical role of platelets in the stimulation of neurons (Kopeikina et al., 2020).

\section{MULTIPLE SCLEROSIS}

Altered platelet function is also suggested in autoimmune neuroinflammatory CNS diseases, such as multiple sclerosis (MS), where the level of activated platelets in the blood of patients is elevated (Morel et al., 2017). MS is an autoimmune disease of the nervous system (CNS) that affects predominantly young adults leading to substantial neurological disability that include upper/lower motor syndrome. MS and experimental autoimmune encephalitis (EAE; an animal model for MS) involve autoimmune Th1 and Th17 cells that recognize myelin self-antigen such as MBP, MOG, and PLP. MS onset usually occurs with the relapsing-remitting type (RRMS), which is characterized by multiple relapses followed by spontaneous remission. Platelets were found to aggravate EAE (Langer et al., 2012; Sotnikov et al., 2013) and induce the development of gray matter damage (Sonia D'Souza et al., 2018). During the early stages of EAE platelets entered the hippocampus, and this is linked with the establishment of a neuroinflammatory environment because of platelet-neuron associations, but not with inflammatory cell infiltration, emphasizing the key role of platelets at the preclinical stages of the disease (Kocovski et al., 2019). This effect along with enhanced anxiety-like behavior observed in mice with EAE was ameliorated after the platelet 
depletion in mice. We have previously shown that plateletderived 5-HT and PAF (Table 1) boosts the differentiation of pathogenic Th1 and Th17 cells during the early stages of MS and EAE (Ponomarev, 2018), while at later stages of the disease, platelets become depleted in granule content but upregulate adhesion molecules such as CD62P to form aggregates with lymphocytes (Starossom et al., 2015). This, in turn, implies that platelets act as a target to alleviate MS symptoms during relapses, among which are subsequent neuropsychiatric symptoms present in these patients (Kocovski et al., 2019). Targeting platelets would be especially effective during the remission period for prophylaxis of future relapses in RRMS. Thus, platelet-neuronal interaction plays an important role during the early stages of MS/EAE and neuronal dysfunctions, which could especially be important for the prevention of future relapses.

\section{PARKINSON'S DISEASE}

The role of platelets in Parkinson's disease (PD) is currently unknown. However, there is accumulating knowledge that suggests the possible involvement of platelets in PD pathology. $\mathrm{PD}$ is a degenerative disorder caused by the loss of dopaminergic neurons in the substantia nigra, which leads to a decrease in motor and cognitive functions. There is no final answer to what causes PD, though mitochondrial dysfunction is implied to be one of the major reasons. A toxin 1-methyl-4-phenyl1,2,3,6-tetrahydropyridine (MPTP), which is selectively toxic for dopaminergic neurons, was found to act by inhibiting complex I of the electron transport chain in neuronal mitochondria, which is important for oxidative phosphorylation. It is known that MPTP is widely used as a chemical intermediate for herbicide production, that could contaminate agricultural products and predispose them to PD (Fukuda, 2001). In addition to herbicides, it was found that insecticide rotenone is also toxic for neurons in the substantia nigra affecting complex I in neuronal mitochondria leading to the symptoms of PD (Sherer et al., 2003).

Decreased complex I activity was found in the lymphocytes and platelets isolated from PD patients when compared to healthy controls (Haas et al., 1995; Subrahmanian and LaVoie, 2021). Moreover, a hybrid PD model where mitochondrial DNA from PD platelets was expressed in human teratocarcinoma cells demonstrated decreased complex I activity in mitochondria of these cells. Besides, 1-methyl-4-phenyl-pyridinium ion $\left(\mathrm{MPP}^{+}\right)$, the metabolite of MPTP, was proved to deplete adenosine triphosphate in platelets and induce attenuated platelet aggregation and activity, which is a probable mechanism of the anti-aggregation effect found in PD patients (Koçer et al., 2013; Leiter and Walker, 2020). Monoamine oxidase $\mathrm{B}$ (MAO-B) enzyme, which is abundant in neuronal and platelet mitochondria, also contributes significantly to MPTP toxicity and the etiology of PD as demonstrated by several studies. Enhanced MAO-B activity has been discovered in PD patients, nevertheless, the findings regarding platelet MAO-B activity in $\mathrm{PD}$ patients are not straightforward enough, as other works imply that platelet MAO-B activity is unaffected in PD patients (Bonuccelli et al., 1990). Nevertheless, the ability of platelets to modulate the functions of mitochondria in the CNS by enhancing oxidative phosphorylation and oxidative stress (Kopeikina et al., 2020) could play an important role in PD pathology.

Besides modulation of neuronal mitochondrial functions by platelets, these cells could affect dopaminergic neurons by producing several substances. Among these factors are a plateletactivating factor (PAF; Table 1), which is produced by activated platelets and plays a pro-inflammatory role in the development of TBI-associated neuroinflammation affecting astrocytes and microglia (Yin et al., 2017; Dukhinova et al., 2018). In addition to glial cells, the PAF receptor (PAFR) is also expressed in neuronal synapses. Stimulation of neurons via PAFR enhanced long-term potentiation and synaptic vesicle release (Hammond et al., 2016). On the other hand, overstimulation of neurons by PAF caused neuronal apoptosis, although the role of PAFR in this process remains controversial (Bennett et al., 1998; Ryan et al., 2007). Mice deficient in PAFR were found to be resistant to the development of disease in a mouse model of MPTP-induced PD. Thus, platelet-derived factors such as PAF could significantly contribute to the development of PD.

\section{PLATELETS AS MARKERS FOR DIAGNOSTICS}

Recently, platelets have come to be regarded as substantial indicators for neurologic diseases of various types. Being multifunctional blood anucleated cells, they are now seen as crucial clinical targets for many neurologic disease pathophysiology. Not only do platelets play a key role in normal hemostasis and thrombosis, but they also are first responders in inflammatory processes (Sotnikov et al., 2013; Starossom et al., 2015; Ponomarev, 2018). They are also involved in a vast range of inflammation-associated pathologies, such as atherosclerosis, cardiovascular diseases, cancer metastasis, and neurodegenerative disorders (Koçer et al., 2013; Franco et al., 2015; Pluta et al., 2018).

During many types of neurodegenerative diseases such as TBI, $\mathrm{AD}$, and $\mathrm{MS}$, platelets change their phenotype and content (Kumar, 2013; Koc et al., 2014; Starossom et al., 2015; Ponomarev, 2018). For example, during AD, platelets show an activated phenotype and there are changes in the processing of platelet $\mathrm{A} \beta$, which could serve as an early marker of AD (Di Luca et al., 1998; Koc et al., 2014). In the mouse model of AD when human APP with Swedish mutation is expressed in the CNS, platelets start to express MMP2 and MMP-9 and damage brain blood vessels in the brain slice model (Kniewallner et al., 2018). This data indicates that CNS-derived amyloid could activate platelets that could contribute to increased BBB permeability. Our data in the epilepsy model also demonstrated that platelets contribute to increased BBB permeability (Kopeikina et al., 2020). In MS, platelets decrease their level of serotonin in dense granules and the level of PF4 in $\alpha$-granules and upregulate adhesion molecules such as CD62P (Starossom et al., 2015). Quite interestingly the exhaustion of platelet granule content and upregulation of CD62P make platelets anti-inflammatory indicating that platelets play a differential role in the early and 


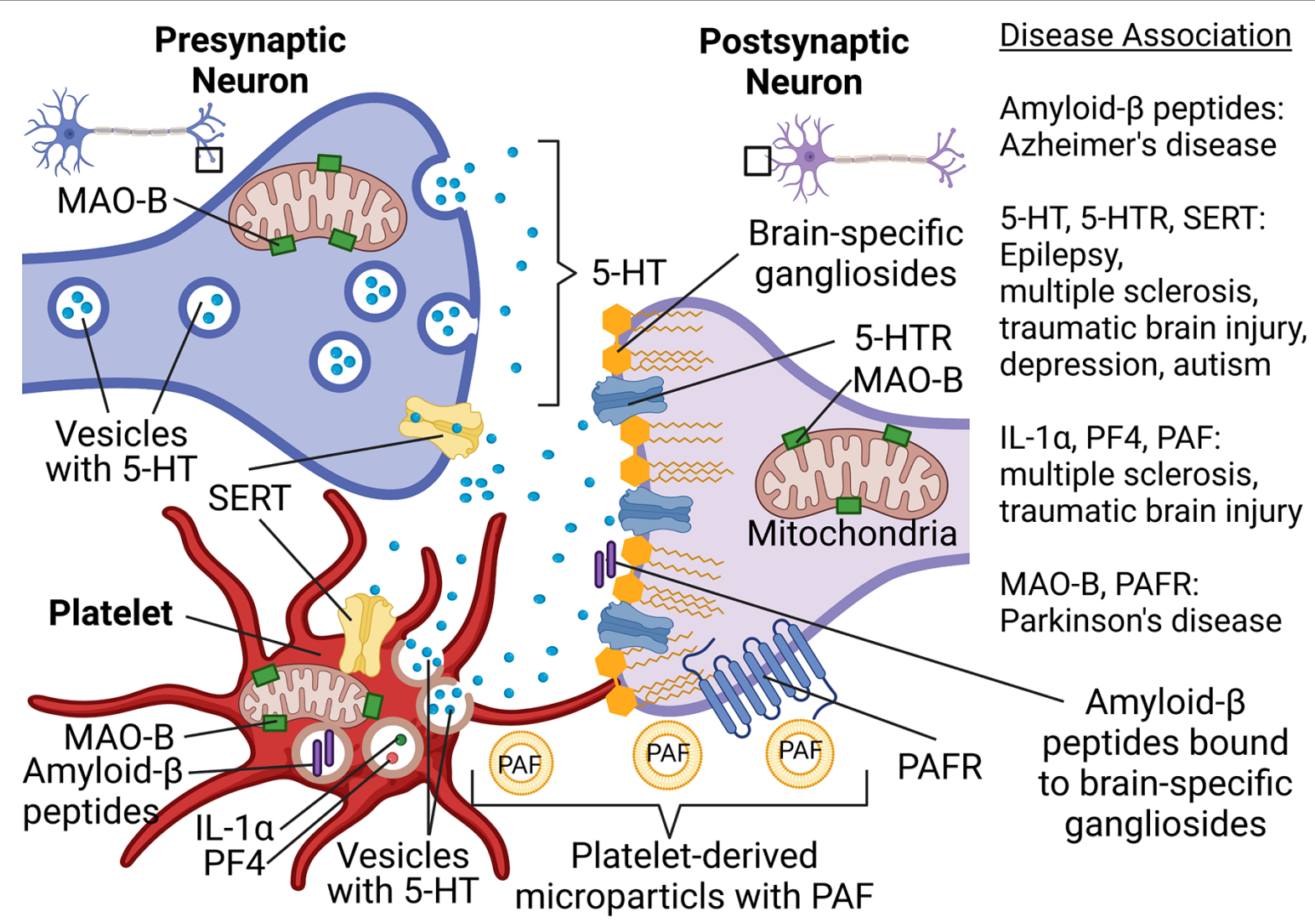

FIGURE 1 | Platelet-neuron similarity and platelet-neuron interactions have implications for a wide range of neuropsychological disorders such as Alzheimer's disease, Parkinson's disease, multiple sclerosis, traumatic brain epilepsy, and depression. Abbreviations: 5-HT, Serotonin; 5-HTR, 5-HT receptor; IL-1 $\alpha$, Interleukin $1 \alpha$; MAO-B, Monoamine oxidase B; PAF, Platelet-activating factor; PAFR, PAF receptor; PF4, Platelet factor-4; SERT, Serotonin transporter. Created in BioRender.com.

late stages of MS (Starossom et al., 2015). This indicates the importance of looking at various parameters to characterize detrimental and beneficial phenotypes of activated platelets to target pathogenic platelet subsets. Imaging cytometry has great potential to assess platelet content such as microRNA (Ponomarev et al., 2011). However, currently, it is challenging, especially in the case of neurologic disorders.

To better understand the possible involvement of platelets in neurologic disorders, a new concept was proposed by several scientists several years ago (Ponomarev, 2018). According to this concept, platelets can be viewed as "circulating mirrors" of neurons and innate immune cells (Ponomarev, 2018; Canobbio, 2019; Figure 1). In the coming years, the analysis of platelet morphology, functionality, metabolism, as well as protein and lipid composition may advance the investigation of neurodegenerative diseases (Figure 1). For example, analysis of metabolism and secretion of serotonin could be used for the understanding of predisposition and progression of depression and TBI. Platelets also use serotonin transporter (SERT), which is targeted by selective serotonin reuptake inhibitors, and anti-depressant drugs (Figure 1). Moreover, we recently demonstrated an important role of serotonin in epilepsy and the formation of new synapses. The latter also plays a role in the development of autism spectrum disorder. The ability of platelets to store, process, and secrete various forms of $A \beta$ could be used as a marker for $\mathrm{AD}$. Analysis of platelets' mitochondrial function could be used to diagnose predisposition for PD (Figure 1). Thus, platelets can become a suitable tool for the analysis of peripheral biomarkers when it comes to diagnosing neuronal dysfunctions in the future (Ehrlich, 2012; Oji et al., 2018; Canobbio, 2019; Padmakumar et al., 2019).

\section{PLATELETS AS TARGETS FOR FUTURE THERAPY}

Targeting platelets and their products for therapeutic purposes in the field of neurological pathologies seems to present an exceptional challenge, both because of the blood-brain barrier and due to obstacles for the drugs on their way to the desired locations of action inside the CNS parenchyma. Above all else, there might be demand for various approaches on how to target platelet or plateletderived microparticles in circulation and CNS parenchyma in the same patients. These interventions will require advanced drug formulations and delivery techniques, along with modern imaging methods to determine drug stability, 
accurate targeting, and efficacy. Antiplatelet therapy is broadly applied in the treatment and prevention of patients with thrombotic cerebrovascular or cardiovascular diseases. Platelet aggregation and activation can be decreased by antiplatelet therapy with one or several drugs available on the market with the most common being heparin and aspirin (Gianazza et al., 2020). There are several antiplatelet drugs currently available on the market with the most popular being aspirin (Chandrasekhara et al., 2016). In the 1960s aspirin appeared as the first valid antiplatelet drug and is still applied to prevent cardiovascular pathologies.

Aspirin was found to ameliorate spontaneous recurrent seizures in SWR/J mice with pilocarpine-induced chronic temporal lobe epilepsy, however, the role of platelets was not investigated in this study (Zhu et al., 2017). It was an interesting case study documenting that woman with epilepsy history who constantly took aspirin (75 mg daily for 3 years) had epileptic convulsion 3 weeks after aspirin withdrawal, but not during the period when she took aspirin (Scheepers et al., 2007). A preliminary clinical study also showed decreased seizure frequency for epilepsy patients on day 2 after starting to take aspirin. The authors of this study emphasize that further prospective study is needed towards this direction (Godfred et al., 2013). Thus, the usage of aspirin (and possibly other antiplatelet drugs) is a perspective for the prevention and treatment of epilepsy. At present, the main platelet target which is used as a target in antiplatelet therapy is cyclooxygenase-1/2 (COX-1/2) with more selective targeting of COX-1. COX-1 is responsible for regulating the production of the prostaglandins, which control platelet aggregation and activation, and is affected by irreversible cyclooxygenase inhibitors such as NSAIDs. NSAIDs were found to be promising for prophylaxis of $\mathrm{AD}$; however, these drugs decrease neuroinflammation by targeting endothelial cells and macrophages and it is not entirely platelet specific (Zhang et al., 2018). Another currently approved more specific antiplatelet drug clopidogrel or its analogs ( $\mathrm{P}^{2} \mathrm{Y}_{12}$ inhibitors) target $\mathrm{ADP}$ receptors on platelets significantly reducing their activation (Amin et al., 2017). Usage of this drug for the prevention and treatment of neurodegenerative diseases is very limited; however, it was recently reported that clopidogrel reduced pathology in the rat model of AD (Khalaf et al., 2020). New drugs and their platelet targets are still being studied, and some promising targets are at the stage of clinical investigation to discover new possible biomarkers for accurate treatment (Coppinger et al., 2007; Yousuf and Bhatt, 2011; Dovizio et al., 2014).

Knowing the important role of platelets in neurodegenerative diseases, it is time to rethink the mechanism of action of some of the known drugs widely used for the treatment of neurologic conditions. There are several candidates for re-evaluation of their therapeutic mechanisms. A common drug for MS called glatiramer acetate (Copaxone ${ }^{\mathrm{TM}}$ ) in addition to targeting $\mathrm{T}$ cells also targets platelets inhibiting their calcium influx, activation, aggregation, and prolongs bleeding time (Starossom et al., 2014). valproic acid, a common drug for the treatment of epilepsy and bipolar disorder, could also affect platelet functions by decreasing their numbers causing thrombocytopenia. So these two drugs could be also called anti-platelet agents.
Most current platelet-specific drugs non-specifically inhibit platelet activation and aggregation, which may cause dangerous complications such as bleeding. Moreover, in certain neurodegenerative diseases, platelets' granule content becomes exhausted and their ability to get further activated is decreased (Starossom et al., 2015), which may require platelet functions to be restored and pathogenic platelets to be replaced by non-pathogenic platelets. To do so, there is a possibility of using autologous genetically engineered iPSC-derived platelets, or artificial platelet-like microparticles for transfusion of patients with neurological disorders (Brown et al., 2014; Moreau et al., 2016; Lawrence et al., 2019). Yet, a more detailed investigation of detrimental vs. beneficial pathways of platelet activation during neurodegenerative diseases should be performed to understand specific pathways in platelets to be targeted using these novel technologies.

\section{CONCLUDING REMARKS}

Recent research results demonstrated that platelets play a crucial role in the pathology of multiple neurologic disorders. During many types of neurodegenerative diseases, the BBB is compromised, which enables platelets to infiltrate the central nervous system (CNS), where they release serotonin and other mediators. Enhanced neuronal electric activity and seizure severity have been greatly contributed to by platelet-derived (but CNS-derived) serotonin and possibly other platelet-derived co-factors. Besides, a whole-genome transcriptome analysis suggested that platelets trigger the expression of many genes associated with neuronal synaptic activity, neuroinflammation, and oxidative phosphorylation in neuronal mitochondria. Based on this, we introduced the model shown in Table 1. Our model implies that platelets could provide the "missing link" between TBI and epilepsy, or initial neuronal damage and further development of other neurodegenerative pathologies such as $\mathrm{AD}, \mathrm{PD}$, and MS (Figure 1). Thus, the main conclusion of our review is that platelets cannot be any further ignored in modern neurobiology.

\section{AUTHOR CONTRIBUTIONS}

EK and EP conceived and wrote the manuscript. EP edited the manuscript. EK made Figure 1. EP made Table 1. All authors contributed to the article and approved the submitted version.

\section{FUNDING}

This work was supported by the Research Grant Council-General Research Fund grant (reference no. 14113316, Hong Kong Government, Hong Kong), by the Research Grant Council-Areas of Excellence Fund grant (reference no. AoE/M604/16, Hong Kong Government, Hong Kong), and by the School of Biomedical Sciences Incentive Scheme-Bridging Fund grant [reference no. SBS-BF(2019-2020)-01 from School of Biomedical Sciences, Chinese University of Hong Kong, Hong Kong]. 


\section{REFERENCES}

Amin, A. M., Sheau Chin, L., Azri Mohamed Noor, D., Sk Abdul Kader, M. A., Kah Hay, Y., Ibrahim, B., et al. (2017). The personalization of clopidogrel antiplatelet therapy: the role of integrative pharmacogenetics and pharmacometabolomics. Cardiol. Res. Pract. 2017:8062796. doi: $10.1155 / 2017 / 8062796$

Au, A. E. L., Sashindranath, M., Borg, R. J., Kleifeld, O., Andrews, R. K., Gardiner, E. E., et al. (2014). Activated platelets rescue apoptotic cells via paracrine activation of EGFR and DNA-dependent protein kinase. Cell Death Dis. 5:e1410. doi: 10.1038/cddis.2014.373

Bennett, S. A. L., Chen, J., Pappas, B. A., Roberts, D. C. S., and Tenniswood, M. (1998). Platelet activating factor receptor expression is associated with neuronal apoptosis in an in vivo model of excitotoxicity. Cell Death Differ. 5, 867-875. doi: $10.1038 /$ sj.cdd.4400434

Bonuccelli, U., Piccini, P., Del Dotto, P., Muratorio, A., Pacifici, G. M., and Corsini, G. U. (1990). Platelet monoamine oxidase B activity in Parkinsonian patients. J. Neurol. Neurosurg. Psychiatry 53, 854-855. doi: 10.1136/jnnp.53.10. 854

Born, H. A. (2015). Seizures in Alzheimer's disease. Neuroscience 286, 251-263. doi: 10.1016/j.neuroscience.2014.11.051

Brown, A. C., Stabenfeldt, S. E., Ahn, B., Hannan, R. T., Dhada, K. S., Herman, E. S., et al. (2014). Ultrasoft microgels displaying emergent platelet-like behaviours. Nat. Mater. 13, 1108-1114. doi: 10.1038/nmat4066

Bu, X. L., Xiang, Y., Jin, W. S., Wang, J., Shen, L. L., Huang, Z. L., et al. (2018). Blood-derived amyloid- $\beta$ protein induces Alzheimer's disease pathologies. Mol. Psychiatry 23, 1948-1956. doi: 10.1038/mp.2017.204

Busche, M. A., Eichhoff, G., Adelsberger, H., Abramowski, D., Wiederhold, K. H., Haass, C., et al. (2008). Clusters of hyperactive neurons near amyloid plaques in a mouse model of Alzheimer's disease. Science 321, 1686-1689. doi: 10.1126/science.1162844

Canobbio, I. (2019). Blood platelets: circulating mirrors of neurons? Res. Pract. Thromb. Haemost. 3, 564-565. doi: 10.1002/rth2.12254

Carhart-Harris, R. L., and Nutt, D. J. (2017). Serotonin and brain function: a tale of two receptors. J. Psychopharmacol. 31, 1091-1120. doi: 10.1177/0269881117725915

Chandrasekhara, V., Chathadi K, V., Early, D. S., Eloubeidi, M. A., Evans, J. A., Faulx, A. L., et al. (2016). The management of antithrombotic agents for patients undergoing GI endoscopy. Gastrointest. Endosc. 83, 3-16. doi: 10.1016/j.gie.2015.09.035

Coppinger, J. A., O’Connor, R., Wynne, K., Flanagan, M., Sullivan, M., Maguire, P. B., et al. (2007). Moderation of the platelet releasate response by aspirin. Blood 109, 4786-4792. doi: 10.1182/blood-2006-07-038539

Cuddapah, V. A., Zhang, S. L., and Sehgal, A. (2019). Regulation of the bloodbrain barrier by circadian rhythms and sleep. Trends Neurosci. 42, 500-510. doi: 10.1016/j.tins.2019.05.001

Devinsky, O., Vezzani, A., O’Brien, T. J., Jette, N., Scheffer, I. E., De Curtis, M., et al. (2018). Epilepsy. Nat. Rev. Dis. Primers 4:18024. doi: 10.1038/nrdp. 2018.24

Di Luca, M., Pastorino, L., Bianchetti, A., Perez, J., Vignolo, L. A., Lenzi, G. L., et al. (1998). Differential level of platelet amyloid $\beta$ precursor protein isoforms: an early marker for Alzheimer disease. Arch. Neurol. 55, 1195-1200. doi: 10.1001/archneur.55.9.1195

Dovizio, M., Alberti, S., Guillem-Llobat, P., and Patrignani, P. (2014). Role of platelets in inflammation and cancer: novel therapeutic strategies. Basic Clin. Pharmacol. Toxicol. 114, 118-127. doi: 10.1111/bcpt.12156

Dukhinova, M., Kuznetsova, I., Kopeikina, E., Veniaminova, E., Yung, A. W. Y., Veremeyko, T., et al. (2018). Platelets mediate protective neuroinflammation and promote neuronal plasticity at the site of neuronal injury. Brain Behav. Immun. 74, 7-27. doi: 10.1016/j.bbi.2018.09.009

Dukhinova, M., Veremeyko, T., Amanda, W. Y. Y., Kuznetsova, I. N. S., Lau, T. Y. B., Kopeikina, E., et al. (2019). Fresh evidence for major brain gangliosides as a target for the treatment of Alzheimer's disease. Neurobiol. Aging 77, 128-143. doi: 10.1016/j.neurobiolaging.2019.01.020

Ehrlich, D. (2012). Platelets in psychiatric disorders. World J. Psychiatry 2:91. doi: 10.5498/wjp.v2.i6.91

Espinosa-Parrilla, Y., Gonzalez-Billault, C., Fuentes, E., Palomo, I., and Alarcón, M. (2019). Decoding the role of platelets and related microRNAs in aging and neurodegenerative disorders. Front. Aging Neurosci. 10:151. doi: 10.3389/fnagi.2019.00151

Fang, W., Geng, X., Deng, Y., Li, Y., Shang, E., Cen, J., et al. (2011). Platelet activating factor induces blood brain barrier permeability alteration in vitro. J. Neuroimmunol. 230, 42-47. doi: 10.1016/j.jneuroim.2010.08.015

Franco, A. T., Corken, A., and Ware, J. (2015). Platelets at the interface of thrombosis, inflammation and cancer. Blood 126, 582-588. doi: 10.1182/blood2014-08-531582

Fukuda, T. (2001). Neurotoxicity of MPTP. Neuropathology 21, 323-332. doi: 10.1046/j.1440-1789.2001.00402.x

Gharedaghi, M. H., Seyedabadi, M., Ghia, J. E., Dehpour, A. R., and Rahimian, R. (2014). The role of different serotonin receptor subtypes in seizure susceptibility. Exp. Brain Res. 232, 347-367. doi: 10.1007/s00221-0133757-0

Gianazza, E., Brioschi, M., Baetta, R., Mallia, A., and Banfi, C. (2020). Platelets in healthy and disease states: from biomarkers discovery to drug targets identification by proteomics. Int. J. Mol. Sci. 21:4541. doi: 10.3390/ijms21124541

Glushakov, A. V., Glushakova, O. Y., Doré, S., Carney, P. R., and Hayes, R. L. (2016). "Animal models of posttraumatic seizures and epilepsy," in Methods in Molecular Biology, (Totowa, NJ: Humana Press Inc.), 481-519.

Godfred, R. M., Parikh, M. S., Haltiner, A. M., Caylor, L. M., Sepkuty, J. P., Doherty, M. J., et al. (2013). Does aspirin use make it harder to collect seizures during elective video-EEG telemetry? Epilepsy Behav. 27, 115-117. doi: 10.1016/j.yebeh.2012.12.031

Gonzales, A. L., Klug, N. R., Moshkforoush, A., Lee, J. C., Lee, F. K., Shui, B., et al. (2020). Contractile pericytes determine the direction of blood flow at capillary junctions. Proc. Natl. Acad. Sci. U S A 117, 27022-27033. doi: 10.1073/pnas. 1922755117

Haas, R. H., Nasirian, F., Nakano, K., Ward, D., Pay, M., Hill, R., et al. (1995). Low platelet mitochondrial complex I and complex II/III activity in early untreated parkinson's disease. Ann. Neurol. 37, 714-722. doi: 10.1002/ana.4103 70604

Hammond, J. W., Lu, S.-M., and Gelbard, H. A. (2016). Platelet activating factor enhances synaptic vesicle exocytosis via pkc, elevated intracellular calcium and modulation of synapsin 1 dynamics and phosphorylation. Front. Cell. Neurosci. 9:505. doi: 10.3389/fncel.2015.00505

Hefter, D., Ludewig, S., Draguhn, A., and Korte, M. (2020). Amyloid, APP and electrical activity of the brain. Neuroscientist 26, 231-251. doi: $10.1177 / 1073858419882619$

Inyushin, M., Zayas-Santiago, A., Rojas, L., and Kucheryavykh, L. (2020). On the role of platelet-generated amyloid beta peptides in certain amyloidosis health complications. Front. Immunol. 11:2587. doi: 10.3389/fimmu.2020.571083

Khalaf, N. E. A., El Banna, F. M., Youssef, M. Y., Mosaad, Y. M., Daba, M. H. Y., and Ashour, R. H. (2020). Clopidogrel combats neuroinflammation and enhances learning behavior and memory in a rat model of Alzheimer's disease. Pharmacol. Biochem. Behav. 195:172956. doi: 10.1016/j.pbb.2020.172956

Kitchigina, V. F. (2018). Alterations of coherent theta and gamma network oscillations as an early biomarker of temporal lobe epilepsy and Alzheimer's disease. Front. Integr. Neurosci. 12:36. doi: 10.3389/fnint.2018.00036

Kniewallner, K. M., de Sousa, D. M. B., Unger, M. S., Mrowetz, H., and Aigner, L. (2020). Platelets in amyloidogenic mice are activated and invade the brain. Front. Neurosci. 14:129. doi: 10.3389/fnins.2020.00129

Kniewallner, K. M., Foidl, B. M., and Humpel, C. (2018). Platelets isolated from an Alzheimer mouse damage healthy cortical vessels and cause inflammation in an organotypic ex vivo brain slice model. Sci. Rep. 8, 1-16. doi: 10.1038/s41598018-33768-2

Kniewallner, K. M., Grimm, N., and Humpel, C. (2014). Platelet-derived nerve growth factor supports the survival of cholinergic neurons in organotypic rat brain slices. Neurosci. Lett. 574, 64-69. doi: 10.1016/j.neulet.2014.05.033

Koçer, A., Yaman, A., Niftaliyev, E., Dürüyen, H., Eryilmaz, M., Koçer, E., et al. (2013). Assessment of platelet indices in patients with neurodegenerative diseases: mean platelet volume was increased in patients with Parkinson's disease. Curr. Gerontol. Geriatr. Res. 2013:986254. doi: 10.1155/2013/ 986254

Koc, E. R., Uzar, E., Cirak, Y., Demir, Y. P., and Ilhan, A. (2014). The increase of mean platelet volume in patients with Alzheimer disease. Turk. J. Med. Sci. 44:1060. doi: 10.3906/sag-1212-5 
Kocovski, P., Jiang, X., D'Souza, C., Li, Z., Dang, P., Wang, X., et al. (2019). Platelet depletion is effective in ameliorating anxiety-like behavior and reducing the pro-inflammatory environment in the hippocampus in murine experimental autoimmune encephalomyelitis. J. Clin. Med. 8:162. doi: 10.3390/jcm80 20162

Kopeikina, E., Dukhinova, M., Yung, A. W. Y., Veremeyko, T., Kuznetsova, I. S., Lau, T. Y. B., et al. (2020). Platelets promote epileptic seizures by modulating brain serotonin level, enhancing neuronal electric activity and contributing to neuroinflammation and oxidative stress. Prog. Neurobiol. 188:101783. doi: 10.1016/j.pneurobio.2020.101783

Kress, G. J., Liao, F., Dimitry, J., Cedeno, M. R., FitzGerald, G. A., Holtzman, D. M., et al. (2018). Regulation of amyloid- $\beta$ dynamics and pathology by the circadian clock. J. Exp. Med. 215, 1059-1068. doi: 10.1084/jem.201 72347

Kumar, M. A. (2013). Coagulopathy associated with traumatic brain injury. Curr. Neurol. Neurosci. Rep. 13:391. doi: 10.1007/s11910-013-0391-y

Löscher, W. (2011). Critical review of current animal models of seizures and epilepsy used in the discovery and development of new antiepileptic drugs. Seizure 20, 359-368. doi: 10.1016/j.seizure.2011.01.003

Langer, H. F., Choi, E. Y., Zhou, H., Schleicher, R., Chung, K. J., Tang, Z., et al. (2012). Platelets contribute to the pathogenesis of experimental autoimmune encephalomyelitis. Circ. Res. 110, 1202-1210. doi: 10.1161/CIRCRESAHA.111. 256370

Lawrence, M., Mueller, A., and Ghevaert, C. (2019). Using genome editing to engineer universal platelets. Emerg. Top. Life Sci. 3, 301-311. doi: 10.1042/ETLS20180153

Leiter, O., and Walker, T. L. (2020). Platelets in neurodegenerative conditions-friend or foe? Front. Immunol. 11:747. doi: 10.3389/fimmu. 2020.00747

Liu, D., Lu, H., Stein, E., Zhou, Z., Yang, Y., and Mattson, M. P. (2018). Brain regional synchronous activity predicts tauopathy in $3 \times \mathrm{TgAD}$ mice. Neurobiol. Aging 70, 160-169. doi: 10.1016/j.neurobiolaging.2018.06.016

Lucke-Wold, B. P., Nguyen, L., Turner, R. C., Logsdon, A. F., Chen, Y. W., Smith, K. E., et al. (2015). Traumatic brain injury and epilepsy: Underlying mechanisms leading to seizure. Seizure 33, 13-23. doi: 10.1016/j.seizure.2015. 10.002

Masters, C. L., Bateman, R., Blennow, K., Rowe, C. C., Sperling, R. A., and Cummings, J. L. (2015). Alzheimer's disease. Nat. Rev. Dis. Primers 1:15056. doi: $10.1038 /$ nrdp.2015.56

McKhann, G. M., Wenzel, H. J., Robbins, C. A., Sosunov, A. A., and Schwartzkroin, P. A. (2003). Mouse strain differences in kainic acid sensitivity, seizure behavior, mortality and hippocampal pathology. Neuroscience 122, 551-561. doi: 10.1016/s0306-4522(03)00562-1

Moreau, T., Evans, A. L., Vasquez, L., Tijssen, M. R., Yan, Y., Trotter, M. W., et al. (2016). Large-scale production of megakaryocytes from human pluripotent stem cells by chemically defined forward programming. Nat. Commun. 7:11208. doi: $10.1038 /$ ncomms 11208

Morel, A., Rywaniak, J., Bijak, M., Miller, E., Niwald, M., and Saluk, J. (2017). Flow cytometric analysis reveals the high levels of platelet activation parameters in circulation of multiple sclerosis patients. Mol. Cell Biochem. 430, 69-80. doi: $10.1007 / \mathrm{s} 11010-017-2955-7$

Musto, A. E., Rosencrans, R. F., Walker, C. P., Bhattacharjee, S., Raulji, C. M., Belayev, L., et al. (2016). Dysfunctional epileptic neuronal circuits and dysmorphic dendritic spines are mitigated by platelet-activating factor receptor antagonism. Sci. Rep. 6, 1-16. doi: 10.1038/srep30298

Oji, S., Tomohisa, D., Hara, W., Tajima, T., Suzuki, M., Saito, A., et al. (2018). Mean platelet volume is associated with early neurological deterioration in patients with branch atheromatous disease: involvement of platelet activation. J. Stroke Cerebrovasc. Dis. 27, 1-8. doi: 10.1016/j.jstrokecerebrovasdis.2018.01. 012

Padmakumar, M., Van Raes, E., Van Geet, C., and Freson, K. (2019). Blood platelet research in autism spectrum disorders: in search of biomarkers. Res. Pract. Thromb. Haemost. 3, 566-577. doi: 10.1002/rth2.12239

Pearson-Smith, J. N., and Patel, M. (2017). Metabolic dysfunction and oxidative stress in epilepsy. Int. J. Mol. Sci. 18:2365. doi: 10.3390/ijms181 12365

Pfisterer, U., Petukhov, V., Demharter, S., Meichsner, J., Thompson, J. J., Batiuk, M. Y., et al. (2020). Identification of epilepsy-associated neuronal subtypes and gene expression underlying epileptogenesis. Nat. Commun. 11, 1-19. doi: $10.1038 / \mathrm{s} 41467-020-18752-7$

Pluta, R., Uamek-Kozio, M., Januszewski, S., and Czuczwar, S. J. (2018). Platelets, lymphocytes and erythrocytes from Alzheimer's disease patients: the quest for blood cell-based biomarkers. Folia Neuropathol. 56, 14-20. doi: 10.5114/fn. 2018.74655

Ponomarev, E. D. (2018). Fresh evidence for platelets as neuronal and innate immune cells: their role in the activation, differentiation and deactivation of Th1, Th17 and tregs during tissue inflammation. Front. Immunol. 9:406. doi: 10.3389/fimmu.2018.00406

Ponomarev, E. D., Veremeyko, T., and Barteneva, N. S. (2011). Visualization and quantitation of the expression of microRNAs and their target genes in neuroblastoma single cells using imaging cytometry. BMC Res. Notes 4:517. doi: 10.1186/1756-0500-4-517

Rainesalo, S., Keränen, T., Saransaari, P., and Honkaniemi, J. (2005). GABA and glutamate transporters are expressed in human platelets. Mol. Brain Res. 141, 161-165. doi: 10.1016/j.molbrainres.2005.08.013

Reed, G. L., Fitzgerald, M. L., and Polgar, J. (2000). Molecular mechanisms of platelet exocytosis: Insights into the "secrete" life of thrombocytes. Blood 96, 3334-3342. doi: 10.1182/blood.V96.10.3334

Ryan, S. D., Harris, C. S., Mo, F., Lee, H., Hou, S. T., Bazan, N. G., et al. (2007). Platelet activating factor-induced neuronal apoptosis is initiated independently of its G-protein coupled PAF receptor and is inhibited by the benzoate orsellinic acid. J. Neurochem. 103, 88-97. doi: 10.1111/j.1471-4159.2007. 04740.x

Scheepers, M., Pearson, A., and Michaelides, M. (2007). Epileptic convulsion following aspirin withdrawal before lid surgery. Eye 21:446. doi: 10.1038/sj.eye. 6702618

Sherer, T. B., Betarbet, R., Testa, C. M., Seo, B. B., Richardson, J. R., Kim, J. H., et al. (2003). Mechanism of toxicity in rotenone models of Parkinson's disease. J. Neurosci. 23, 10756-10764. doi: 10.1523/JNEUROSCI.23-34-107 56.2003

Sonia D'Souza, C., Li, Z., Luke Maxwell, D., Trusler, O., Murphy, M., Crewther, S., et al. (2018). Platelets drive inflammation and target gray matter and the retina in autoimmune-mediated encephalomyelitis. J Neuropathol. Exp. Neurol. 77, 567-576. doi: 10.1016/j.ymthe.2021.01.033

Sotnikov, I., Veremeyko, T., Starossom, S. C., Barteneva, N., Weiner, H. L., and Ponomarev, E. D. (2013). Platelets recognize brain-specific glycolipid structures, respond to neurovascular damage and promote neuroinflammation. PLoS One 8:e58979. doi: 10.1371/journal.pone.00 58979

Staley, K. (2015). Molecular mechanisms of epilepsy. Nat. Neurosci. 18, 367-372. doi: $10.1038 / \mathrm{nn} .3947$

Starossom, S. C., Veremeyko, T., Dukhinova, M., Yung, A. W. Y., and Ponomarev, E. D. (2014). Glatiramer acetate (Copaxone) modulates platelet activation and inhibits thrombin-induced calcium influx: possible role of copaxone in targeting platelets during autoimmune neuroinflammation. PLoS One 9:e96256. doi: 10.1371/journal.pone.0096256

Starossom, S. C., Veremeyko, T., Yung, A. W. Y., Dukhinova, M., Au, C., Lau, A. Y., et al. (2015). Platelets play differential role during the initiation and progression of autoimmune neuroinflammation. Circ. Res. 117, 779-792. doi: 10.1161/CIRCRESAHA.115.306847

Subrahmanian, N., and LaVoie, M. J. (2021). Is there a special relationship between complex I activity and nigral neuronal loss in Parkinson's disease? a critical reappraisal. Brain Res. 2021:147434. doi: 10.1016/j.brainres.2021. 147434

Thijs, R. D., Surges, R., O'Brien, T. J., and Sander, J. W. (2019). Epilepsy in adults. Lancet 393, 689-701. doi: 10.1016/S0140-6736(18) 32596-0

Tobergte, D. R., and Curtis, S. (2013). Neuropsychiatric disorders. An integrative approach. J. Chem. Inf. Model 53, 1689-1699.

Tomkins, O., Shelef, I., Kaizerman, I., Eliushin, A., Afawi, Z., Misk, A., et al. (2008). Blood-brain barrier disruption in post-traumatic epilepsy. J. Neurol. Neurosurg. Psychiatry 79, 774-777. doi: 10.1136/jnnp.2007. 126425

Veitinger, M., Varga, B., Guterres, S. B., and Zellner, M. (2014). Platelets, a reliable source for peripheral Alzheimer's disease biomarkers? Acta Neuropathol. Commun. 2:65. doi: 10.1186/2051-5960-2-65 
Wu, T., Chen, L., Zhou, L., Xu, J., and Guo, K. (2021). Platelets transport $\beta$-amyloid from the peripheral blood into the brain by destroying the blood-brain barrier to accelerate the process of Alzheimer's disease in mouse models. Aging (Albany NY) 13, 7644-7659. doi: 10.18632/aging.202662

Yamamoto, H., and Gurney, M. E. (1990). Human platelets contain brain-derived neurotrophic factor. J. Neurosci. 10, 3469-3478. doi: 10.1523/JNEUROSCI.10$11-03469.1990$

Yin, X. J., Chen, Z. Y., Zhu, X. N., and Hu, J. J. (2017). Loss of PAFR prevents neuroinflammation and brain dysfunction after traumatic brain injury. Sci. Rep. 7, 1-12. doi: 10.1038/srep40614

Yousuf, O., and Bhatt, D. L. (2011). The evolution of antiplatelet therapy in cardiovascular disease. Nat. Rev. Cardiol. 8, 547-559. doi: 10.1038/nrcardio. 2011.96

Zhang, C., Wang, Y., Wang, D., Zhang, J., and Zhang, F. (2018). NSAID exposure and risk of Alzheimer's disease: an updated meta-analysis from cohort studies. Front. Aging Neurosci. 10:83. doi: 10.3389/fnagi.2018.00083
Zhu, K., Hu, M., Yuan, B., Liu, J. X., and Liu, Y. (2017). Aspirin attenuates spontaneous recurrent seizures in the chronically epileptic mice. Neurol. Res. 39, 744-757. doi: 10.1080/01616412.2017.13 26657

Conflict of Interest: The authors declare that the research was conducted in the absence of any commercial or financial relationships that could be construed as a potential conflict of interest.

Copyright (c) 2021 Kopeikina and Ponomarev. This is an open-access article distributed under the terms of the Creative Commons Attribution License (CC BY). The use, distribution or reproduction in other forums is permitted, provided the original author(s) and the copyright owner(s) are credited and that the original publication in this journal is cited, in accordance with accepted academic practice. No use, distribution or reproduction is permitted which does not comply with these terms. 